\title{
Determining the Impact of Increased Physical Activity on Improving Sleep Quality in Young Adults
}

\author{
Ashley Darling \\ Virginia Commonwealth University \\ Alex Chiu \\ Eric Henderson \\ See next page for additional authors
}

Follow this and additional works at: https://scholarscompass.vcu.edu/gradposters

Part of the Rehabilitation and Therapy Commons

\section{Downloaded from}

Darling, Ashley; Chiu, Alex; Henderson, Eric; Autler, Aaron; Weggen, Jennifer; Decker, Kevin; and Garten, Ryan, "Determining the Impact of Increased Physical Activity on Improving Sleep Quality in Young Adults" (2020). Graduate Research Posters. Poster 82.

https://scholarscompass.vcu.edu/gradposters/82

This Poster is brought to you for free and open access by the Graduate School at VCU Scholars Compass. It has been accepted for inclusion in Graduate Research Posters by an authorized administrator of VCU Scholars Compass. For more information, please contact libcompass@vcu.edu. 


\section{Authors}

Ashley Darling, Alex Chiu, Eric Henderson, Aaron Autler, Jennifer Weggen, Kevin Decker, and Ryan Garten 


\section{Determining the Impact of Increased Physical Activity on Improving Sleep Quality in Young Adults}

A. Darling, A. Chiu, E. Henderson, A. Autler, J. Weggen, K. Decker and R.S. Garten. Dept. of Kinesiology and Health Sciences

Virginia Commonwealth University, Richmond, VA.

\section{ABSTRACT}

Disturbed sleep, defined as any alteration to normal sleep patterns, has been linked to poor cardiovascular health and an increase in cardiovascular disease (CVD) risk. These negative sleep patterns are highly prevalent with $35 \%$ to $41 \%$ of individuals in the United States reported some form of disturbed sleep. Although high amounts of physical activity (PA) are often associated with high sleep quality, little is known about PA's effectiveness to improve different aspects of sleep (e.g. duration vs quality) and the mechanisms to which it can improve sleep quality. PURPOSE: The study sought to determine the ability of increased PA to improve sleep efficiency in healthy young adults. METHODS: Nineteen young adults ( $25 \pm 4$ yrs) were recruited for this study. Subjects wore an accelerometer (Actigraph GT3x-BT) for a total of three weeks to record daily physical activity (step count; moderate-to-vigorous physical activity (MVPA)) and sleep variables (total time in bed (TTB), sleep efficiency, wake after sleep onset (WASO), and total sleep time(TST)). Subjects maintained normal physical activity levels for the first week (BL). then increased their step count by an average of 5,000 steps/day across the next two weeks (W1 and W2). Heart rate variability (HRV) and venous blood draws were collected weekly to assess sympathetic activity and inflammation, respectively. RESULTS: The physical activity intervention resulted in significant increases $(\mathrm{p}<0.001)$ in MVPA and step-count for both W1 $(13163 \pm 3184)$ and W2 $(12168 \pm 3619)$ when compared to BL ( $8648 \pm 2615$ steps/day). No significant differences from BL were observed whe examining sleep efficiency (BL: $83.8 \pm 6.4$; W $1: 85.5 \pm 4.0$; W2: $84.2 \pm 6.1 \%$ ), sympathetic-vagal
balance, and inflammatory marker concentrations in W1 and W2. A significant correlation was revealed when assessing the change in sleep efficiency from BL to W1 $(r=-0.81, p<0.001)$ when compared to Witial sleep efficiency values. CONCLUSION: This study revealed that although young healthy individuals appear to lack improvements in sleep efficiency with an increase in physical activity, those who reported the lowest sleep quality had the greatest improvements in sleep efficiency following an increase in physical activity. Therefore the findings of the study suggest that although increasing physical activity can improve sleep quality, a potential "ceiling effect" may occur, as when sleep quality is actequate, augmenting physica activity no longe has a substial effect.

\section{METHODS}

Subjects

Nineteen part in this study.

Physical Activity Intervention

Subjects increased their step count by an average of 5,000 steps/day for the two weeks following BL measures. A pedometer (Omron HJ-321) was given to each subject to provide real-time, daily visual feedback of their step count

Sleep Measures

Subjects wore an accelerometer (Actigraph GT3x-BT) for a total of three weeks. The accelerometer was won on he righ hip in the midhe of hee high during the day to record PA (step count and MVPA) and worn on the non-dominant wrist during hight to record sleep (TTB, sleep efficiency, WASO, and TST). Autonomic Nervous System and Intammatory Markers

Five minutes of HRV was collected in the supine position after 10 minutes of rest. Venous blood draws were collected to measure inflammatory markers IL-6 and TNF-alpha using commercially available assay kits (R\&D Systems, Minneapolis, MN, USA)

Statistical Analysis

A one-way ANOVA was employed for each normally distributed measure (step count, MVPA, TST, TNF-alpha). A Wilcoxon rank sum test was employed when data sets were not normally distributed (sleep efficiency, WASO, IL-6, LF/HF ratio). Subsequent post hoc analyses (Wilcoxon each pair and Tukey-HSD) were employed when a significant main effect was revealed. Pearson correlations were utilized to determine relationships between sleep variables and physical activity, inflammatory markers, and HRV measures. Level of significance for all tests was set a priori at $\alpha<0.05$.

\section{RESULTS}

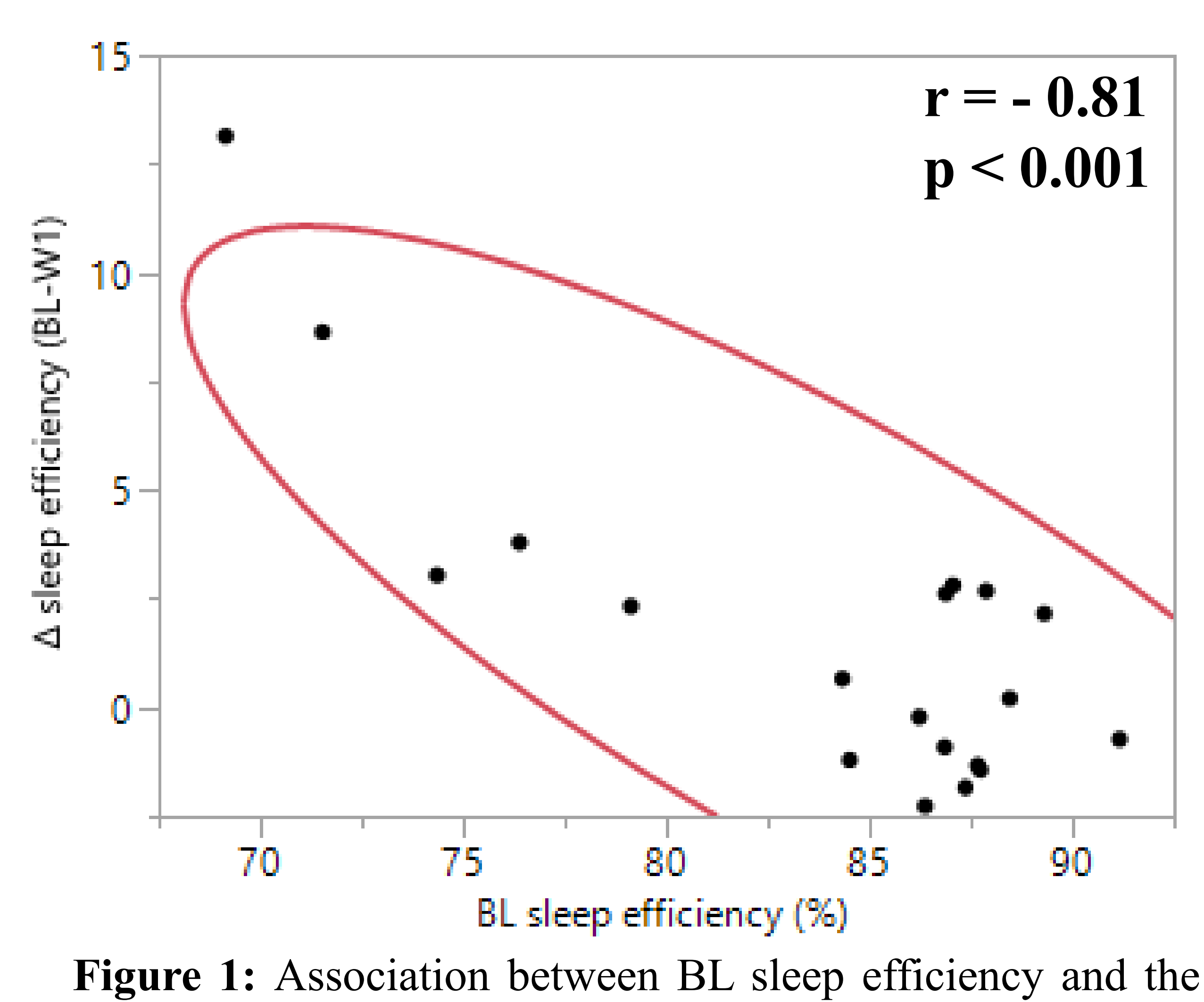

change in sleep efficiency from BL to W1. Density ellipse is set at $95 \%$ probability.

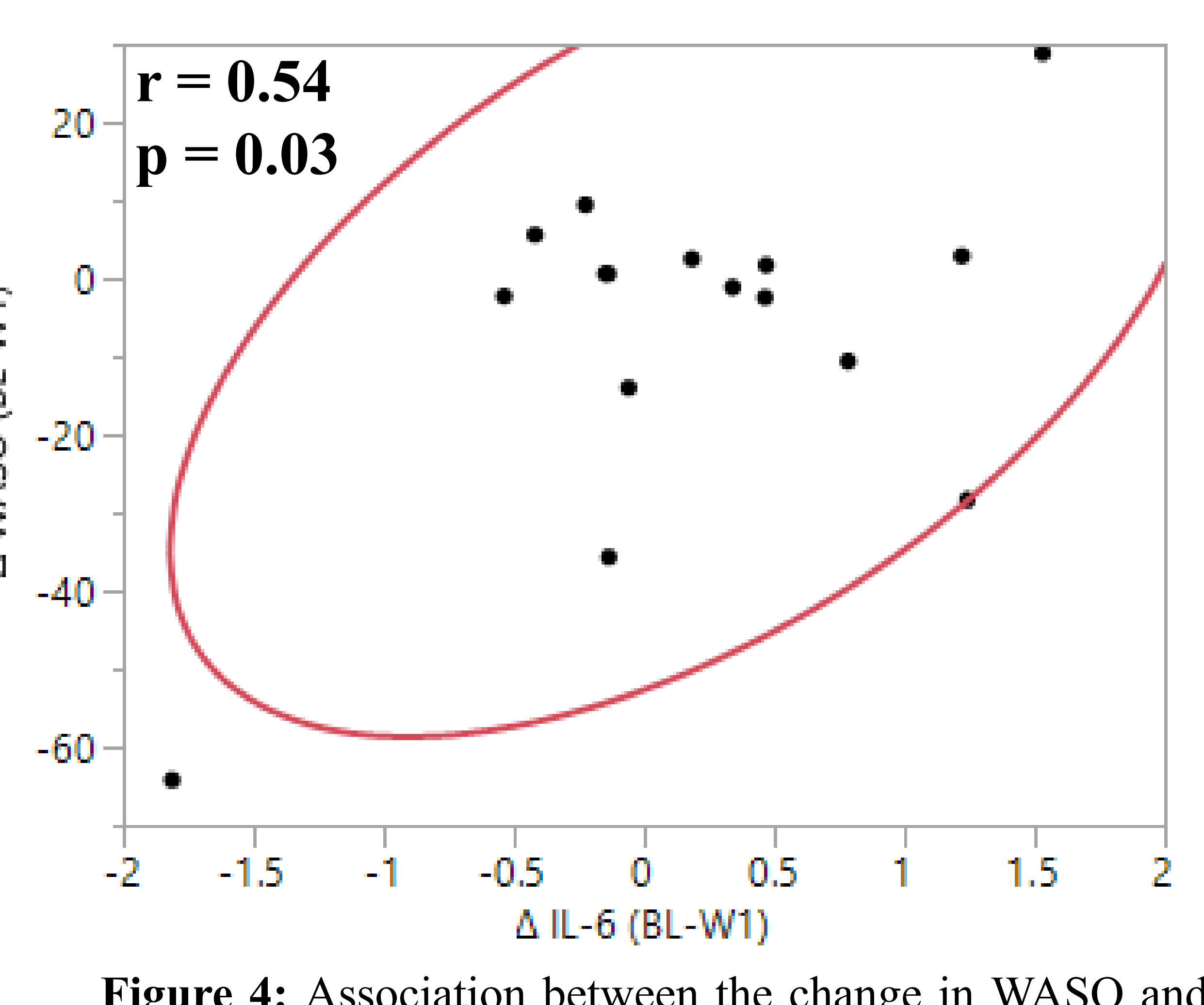

Figure 4: Association between the change in WASO and
IL-6 concentration from BL to W1. Density ellipse is set at $95 \%$ probability

Table 2: Physical activity, sleep, inflammation, and autonomic nervous system (ANS) measures across time 「Mean \pm SD or median $\left(25^{\text {th }}\right.$ percentile, $75^{\text {th }}$ percentile). * $p<0.001$ vs BL

\begin{tabular}{lccc}
\hline \hline Variable & BL & W1 & W2 \\
\hline Physical Activity Measures & & & \\
Step count (average per day) & $8648 \pm 2615$ & $13163 \pm 3184^{*}$ & $12169 \pm 3619^{*}$ \\
MVPA (average minutes/day) & $57 \pm 26$ & $93 \pm 34^{*}$ & $89 \pm 30^{*}$ \\
Sedentary Time (avg minutes/day) & $637 \pm 91$ & $615 \pm 64$ & $610 \pm 59$ \\
Sleep Measures & & & \\
TTB (average minutes/night) & $456 \pm 55$ & $455 \pm 59$ & $470 \pm 69$ \\
Sleep efficiency (\%) & $87(79,88)$ & $86(82,90)$ & $84(81,90)$ \\
WASO (average minutes/night) & $52(43,85)$ & $61(45,72)$ & $64(45,92)$ \\
TST (average minutes/night) & $380 \pm 59$ & $389 \pm 57$ & $396 \pm 63$ \\
Inflammation \& ANS modulation & & & \\
IL-6 (picograms/milliliter) & $0.75(0.71,1.16)$ & $0.72(0.60,1.34)$ & $0.76(0.62,0.83)$ \\
TNF-alpha (picograms/milliliter) & $1.0 \pm 0.40$ & $0.93 \pm 0.28$ & $0.93 \pm 0.33$ \\
Sympathetic-Vagal ratio & $0.99(0.5,1.4)$ & $0.7(0.4,1.2)$ & $0.9(0.3,2.2)$ \\
\hline \hline
\end{tabular}

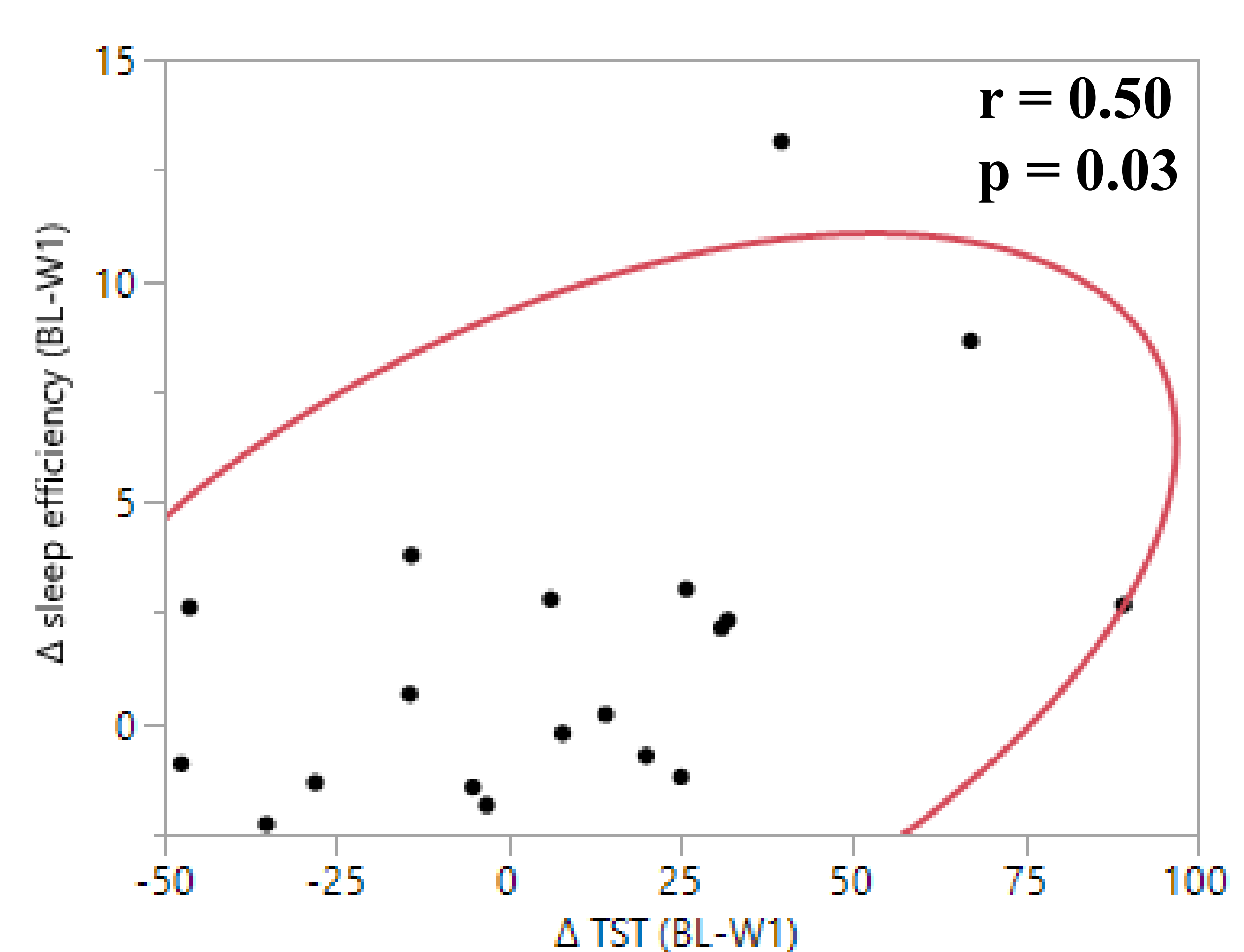

Figure 3: Association between the change in sleep efficiency and TST from BL to W1. Density ellipse is set $95 \%$ probability

Table 1: Subject characteristics. $(\mathrm{n}=19)$

\begin{tabular}{lc}
\hline \hline Subject Characteristics & Mean \pm SD \\
\hline Anthropometric Data & \\
Age (years) & $25 \pm 5$ \\
Weight (kilograms) & $67 \pm 12$ \\
Height (centimeters) & $167 \pm 8$ \\
BMI (kilograms/meters ${ }^{2}$ ) & $25 \pm 3$ \\
Body Fat (\%) & $26 \pm 10$ \\
\hline \hline
\end{tabular}

\section{CONCLUSIONS}

- An increase in PA does not appear to improve sleep quality in healthy young adults (Table 2); however, those who reported the lowest sleep quality had tim incrovements in sleep efficiency following an increase in PA (Figure 1).

Improvements in sleep efficiency following an increase in PA were driven by a decrease in WASO (Figure 2) and an increase in TST (Figure 3)

Improvements in WASO were associated with decreases in IL-6 (Figure 4), and improvements in TST were associated with increases in sympathetic nervous system activity (Figure 5).

- Taken together, increasing PA can improve sleep quality via decreases in inflammation and increases in sympathetic effect" may occur, as augmenting PA no longer has a substantial effect.

Correspondence: rsgarten@vcu.edu 